University of Texas at El Paso

ScholarWorks@UTEP

\title{
Fuzzy Techniques Explain Empirical Power Law Governing Wars and Terrorist Attacks
}

\author{
Hung T. Nguyen \\ New Mexico State University - Main Campus, hunguyen@nmsu.edu \\ Kittawit Autchariyapanitkul \\ Maejo University, kittar3@hotmail.com \\ Vladik Kreinovich \\ The University of Texas at El Paso, vladik@utep.edu
}

Follow this and additional works at: https://scholarworks.utep.edu/cs_techrep

Part of the Computer Sciences Commons

Comments:

Technical Report: UTEP-CS-16-103a

To appear in Proceedings of the Joint 17th Congress of International Fuzzy Systems

Association and 9th International Conference on Soft Computing and Intelligent Systems, Otsu, Japan, June 27-30, 2017.

\section{Recommended Citation}

Nguyen, Hung T.; Autchariyapanitkul, Kittawit; and Kreinovich, Vladik, "Fuzzy Techniques Explain Empirical Power Law Governing Wars and Terrorist Attacks" (2017). Departmental Technical Reports (CS). 1076. https://scholarworks.utep.edu/cs_techrep/1076 


\section{Fuzzy Techniques Explain Empirical Power Law Governing Wars and Terrorist Attacks}

\author{
Hung T. Nguyen \\ Department of Mathematical Sciences \\ New Mexico State University \\ Las Cruces, NM 88003, USA, and \\ Faculty of Economics, Chiang Mai University \\ Chiang Mai, Thailand \\ hunguyen@nmsu.edu
}

\author{
Kittawit Autchariyapanitkul \\ Faculty of Economics \\ Maejo University \\ Chiang Mai, Thailand \\ kittar3@hotmail.com
}

\author{
Vladik Kreinovich \\ Department of Computer Science \\ University of Texas at El Paso \\ $500 \mathrm{~W}$. University \\ El Paso, Texas 79968, USA \\ vladik@utep.edu
}

\begin{abstract}
The empirical distribution of the number of casualties in wars and terrorist attacks follows a power law with exponent 2.5. So far, there has not been a convincing explanation for this empirical fact. In this paper, we show that by using fuzzy techniques, we can explain this exponent. Interesting, we can also get a similar explanation if we use probabilistic techniques. The fact that two different techniques lead to the same explanation makes us reasonably confident that this explanation is correct.
\end{abstract}

\section{EMPIRICAL POWER LAW GOVERNING WARS AND TERRORIST ATTACKS: INTRODUCTION}

For historic data about wars, it is possible to perform statistically reliable analysis. Wars are horrible, people die. Good news is that conflicts with numerous casualties are relatively rare. Bad news that in the history of mankind, there have been so many conflicts with numerous causalities that it is already possible to make statistically significant conclusions based on the historical data.

Empirical power law: a description. One statistical conclusion that researchers have extracted from the analysis of historical data is that the wars follow the power law: namely, if we consider all the conflicts in all geographic areas, then the overall number of conflicts with $n$ casualties decreases with $n$ as $c \cdot n^{-2.5}$; see, e.g., [5].

In other world, the probability density function $\rho(n)$ that describes the distribution of different wars by the number of casualties has, for sufficiently large $n$ (i.e., for $n \geq n_{0}$ for some $\left.n_{0}\right)$, the form

$$
\rho(n)=c \cdot n^{-2.5}
$$

Terrorist attacks follow the same pattern. The original power law was used to describe wars, i.e., events that caused at least 1000 casualties. Later, it turned out that the same power law also accurately describes events with fewer casualties, such as terrorist attacks; see, e.g., [2] and references therein.

But why? While the newly arriving data continues to confirm the power law for wars and terrorist attacks, there is still no 978-1-5090-4917-2/17\$31.00 (C) 2017 IEEE convincing theoretical explanation for the specific exponent 2.5 of the corresponding power law.

What we do in this paper. In this paper, we show that the use of fuzzy techniques can provide an explanation for the observed exponent of the corresponding power law.

Once we came up with this explanation, we raised a natural question: how general is our explanation? Does this explanation depend on the specific features of the fuzzy techniques that we used - or it is general enough to be valid under a general description of uncertainty? To check this, we decided to see what will happen if, to describe the corresponding uncertainty, we consider probabilistic techniques instead of the fuzzy ones. To our pleasant surprise, with probabilistic techniques, we were able to get a similar explanation.

The fact that two different types of techniques leads to the same explanation makes us more confident that this explanation is correct.

\section{General Analysis of the Problem}

Let us consider reasonable constraints on the probability density $\rho(n)$. Our objective is to explain why, of all possible power laws $\rho(n) \sim n^{-\alpha}$, wars and terrorist attacks are described by the exponent $\alpha=2.5$.

To come up with such an explanation, let us first analyze, based on the first principles, which values of the exponent $\alpha$ are reasonable in the description of wars and terrorist attacks.

First idea: the overall expected number of casualties is finite. This sounds trivial from a common sense viewpoint, but this is a serious requirement from a mathematical viewpoint, since for many power laws, the expected value of the corresponding random variable is infinite.

Let us consider this requirement from a mathematical viewpoint. The expected value of the random variable $n$ under the probability distribution described by the probability density function $\rho(n)$ is equal to the integral

$$
\int n \cdot \rho(n) d n
$$


For the power law $\rho(n) \sim n^{-\alpha}$, the above integral becomes proportional to $\int_{n_{0}}^{\infty} n^{1-\alpha} d n$. This integral, in its turn, is proportional to the difference between the value $n^{2-\alpha}$ at the starting point $n_{0}$ and at infinity.

For $n=n_{0}$, the value of $n^{2-\alpha}$ is, of course, finite. So, to make sure that the whole expected value is finite, we need to ensure that the value $n^{2-\alpha}$ becomes finite at infinity. Thus, we cannot have $\alpha<2-$ else this value will be infinite.

We cannot have $\alpha=2$ either - because then, the integral describing the mean takes the form $\int_{n_{0}}^{\infty} n^{-1} d n$, which is equal to $\ln (\infty)-\ln \left(n_{0}\right)$ and thus, to infinity.

So, the natural requirement that the overall expected number of casualties is finite implies that $\alpha>2$.

Second idea: taking into consideration that wars and terrorist attacks are difficult to predict. It is a known commonsense fact that it is very difficult to predict wars or terrorist attacks.

While this statement makes sense from the viewpoint of common sense, from the viewpoint of the corresponding probabilistic model, this may sound somewhat unexpected. Indeed, the whole purpose of statistical data analysis is to make predictions. Yes, sometimes we do not know the probability distribution; in such cases, of course, prediction is difficult. But here, we know exactly the probability distribution - so why is prediction difficult?

The answer lies in the fact that the accuracy of most statistics-based predictions - starting from the simplest possible case of using arithmetic average as an estimate for the mean - is described in terms of the corresponding standard deviation; see, e.g., [6].

- For the usual normal distributions, standard deviations are finite (and usually small), so we can have reasonably accurate predictions.

- However, for many power laws, variance is infinite (hence standard deviation is infinite). So, accurate predictions are not possible.

(That the variance is sometimes infinite is not surprising: as we have mentioned earlier, for the power law, even the means are often infinite.)

In these terms, the common sense idea that wars and terrorist attacks are difficult to predict seems to indicate that for the specific power that describes these phenomena, the variance should be infinite.

Let us describe this requirement in terms of the exponent $\alpha$. The variance $V$ is equal to $M_{2}-\mu^{2}$, where

$$
M_{2} \stackrel{\text { def }}{=} \int n^{2} \cdot \rho(n) d n
$$

is the second moment and $\mu$ is the mean. Thus, in situations when the mean is finite, infiniteness of the variance is equivalent to infiniteness of the second moment.

For a general power law $\rho(n) \sim n^{-\alpha}$, the second moment is proportional to

$$
\int_{n_{0}}^{\infty} n^{2} \cdot n^{-\alpha} d n=\int_{n_{0}}^{\infty} n^{2-\alpha} d n=\left.\frac{n^{3-\alpha}}{3-\alpha}\right|_{n_{0}} ^{\infty} .
$$

The value $n^{3-\alpha}$ is finite for $n=n_{0}$, so for the integral to be infinite, this expression need to be infinite when $n \rightarrow \infty$. Thus, we must have $\alpha<3$.

Conclusion. Our analysis shows that the exponent of the corresponding power law must be between 2 and 3 .

Remaining question. Which value from the interval $(2,3)$ should we choose? Or, stated differently: how can we explain that the empirical data is in very good accordance with one specific value from this interval: namely, the value $\alpha=2.5$ ?

What we do next. In the following section, we show that the use of fuzzy techniques explains the use of 2.5. In the section after that, we show that probabilistic techniques can also explain the use of $\alpha=2.5$.

As we have mentioned, the fact that two different techniques lead to the same conclusion make us confident that this conclusion is indeed true.

\section{LET Us APPly FUZZY TEChNIQUES}

Why fuzzy? At first glance, we have a very precise description of the situation: we have a value $\alpha$ in an interval $(2,3)$. This is a very crisp statement, with no uncertainty, no fuzziness.

However, the situation is not as crisp as it may seem at first glance. From the purely mathematical viewpoint, finiteness of the overall losses means that $\alpha>2$. However, as the value $\alpha$ gets closer and closer to 2 , the expected value becomes larger and larger - and thus, unrealistic. In other words, it is not enough to require that the expected number of losses is finite, we also must require that this expected value is not too large - and thus, that $\alpha$ should be significantly larger than 2 .

Here comes fuzziness: "not too large" is not a precise term, as well as "significantly larger". To describe such terms, fuzzy logic techniques have been invented [7]; see, e.g., [3], [4].

Similarly, instead of a seemingly crisp inequality $\alpha<3$, we have, in reality, a fuzzy inequality.

How to describe this fuzziness in precise terms. We want to make sure that both differences $\alpha-2$ and $3-\alpha$ are positive - in some fuzzy sense.

Let $\mu(x)$ be a membership function that describes this "positiveness". The larger the positive number, the more confident we are that this number is common-sense positive. Thus, the function $\mu(x)$ should be increasing with $x$.

In terms of this membership function, for each $\alpha$, the degree to which the first inequality is satisfied is equal to $\mu(\alpha-2)$, and the degree to which the second inequality is satisfied is equal to $\mu(3-\alpha)$.

To find the degree to which both conditions are satisfied, we must apply an appropriate "and"-operation (t-norm) $f_{\&}(a, b)$ to these two numbers $a=\mu(\alpha-2)$ and $b=\mu(3-\alpha)$ and thus, get the value

$$
f_{\&}(\mu(\alpha-2), \mu(3-\alpha)) .
$$

Based on the above precise description of fuzziness, which value $\alpha$ should we select? The above expression describes the 
degree to which a given value $\alpha$ satisfies both inequalities. If we want to select a single number, a reasonable idea is to select the value $\alpha$ for which we are most confident that this value satisfies both inequalities.

We therefore need to select a value $\alpha$ for which the above expression attains its largest possible value.

From the idea to the actual selection. The resulting selection of $\alpha$, in general, depends of the choice of a membership function $\mu(x)$ and of the "and"-operation $f_{\&}(a, b)$.

Since we have no a priori reason to select one or another "and"-operations, let us select the computationally simplest one $f_{\&}(a, b)=\min (a, b)$. In this case, the optimized function has the form

$$
\min (\mu(\alpha-2), \mu(3-\alpha))
$$

Let us show that this expression attains its maximum when $\alpha=2.5$. Indeed, for this particular value $\alpha$, the above expression takes the form

$$
\min (\mu(0.5), \mu(0.5))=\mu(0.5) .
$$

On the other hand, for any value $\alpha<2.5$, we have

$$
\alpha-2<0.5<3-\alpha .
$$

Thus, due to monotonicity of the membership function $\mu(x)$, we have

$$
\mu(\alpha-2)<\mu(0.5)<\mu(3-\alpha)
$$

and thus,

$$
\min (\mu(\alpha-2), \mu(3-\alpha))=\mu(\alpha-2)<\mu(0.5) .
$$

Similarly, for any value $\alpha>2.5$, we have

$$
3-\alpha<0.5<\alpha-2 .
$$

Thus, due to monotonicity of the membership function $\mu(x)$, we have

$$
\mu(3-\alpha)<\mu(0.5)<\mu(\alpha-2)
$$

and thus,

$$
\min (\mu(\alpha-2), \mu(3-\alpha))=\mu(3-\alpha)<\mu(0.5) .
$$
2.5

So, the maximum is indeed attained only when we have $\alpha=$

Conclusion. Thus, fuzzy idea indeed explain why we should select the exponent $\alpha=2.5$ in the description of statistics of wars and terrorist attacks.

\section{Let Us Apply Probabilistic Techniques}

Analysis of the problem: let us introduce a probability distribution on the interval $(2,3)$. We are uncertain about the value $\alpha$. All we know about the unknown exponent value $\alpha$ is that this value lines somewhere on the interval $(2,3)$.

In the probabilistic approach, to describe this uncertainty, we can use a probability distribution $f(\alpha)$ on the interval $(2,3)$; see, e.g., [6]. Of course, there are many different probability distributions on this interval. Which distribution should we choose?
Let us use Laplace's Indeterminacy Principle. We do not have any reason to believe that some values from this interval are more probable than others. Thus, it make sense to assume that all the values from the interval are equally probable, i.e., that we have a uniform distribution on this interval. This argument - dating back to Laplace, one of the founders of the probability theory - is known as Laplace's Indeterminacy Principle.

Its modern form is known as the Maximum Entropy approach (see, e.g., [1]) when out of all possible probability distributions, we select a one for which the entropy

$$
S \stackrel{\text { def }}{=}-\int f(\alpha) \cdot \ln (f(\alpha)) d \alpha
$$

attains its largest possible value.

For distributions located on the interval $(2,3)$, the only constraint on the value $f(\alpha)$ is that the overall probability to be on this interval should be equal to $1: \int_{2}^{3} f(\alpha) d \alpha=1$.

Thus, we arrive at the following constraint optimization problem: maximize the expression $-\int_{2}^{3} f(\alpha) \cdot \ln (f(\alpha)) d \alpha$ under the constraint $\int_{2}^{3} f(\alpha) d \alpha=1$. By using the Lagrange multiplier method, we can reduce this constraint optimization problem to the following unconstraint one: maximize

$$
-\int_{2}^{3} f(\alpha) \cdot \ln (f(\alpha)) d \alpha+\lambda \cdot\left(\int_{2}^{3} f(\alpha) d \alpha-1\right),
$$

where the constant $\alpha$ must be defined from the condition that the constraint $\int_{2}^{3} f(\alpha) d \alpha=1$ be satisfied.

Differentiating this expression with respect to each unknown $f(\alpha)$ and equating the derivative to 0 , we conclude that

$$
-\ln (f(\alpha))-1+\lambda=0,
$$

i.e., that $\ln (f(\alpha))=\lambda-1$ and thus, that $f(\alpha)=\exp (\lambda-1)$. Thus, $f(\alpha)$ does not depend on $\alpha$, it is a constant $f(\alpha)=\rho_{0}$, for some constant $\rho_{0}$.

The condition $\int_{2}^{3} f(\alpha) d \alpha=1$ now implies that

$$
\int_{2}^{3} \rho_{0} d \alpha=1
$$

and thus, that $\rho_{0}=1$. So, we indeed have a uniform distribution on the interval $(2,3)$.

So what value from the interval $(2,3)$ should we choose: a problem. Now we know the reasonable probability distribution on the set of all possible values $\alpha$ - namely, the uniform distribution, with probability density $f(\alpha)=1$.

Based on this distribution, which value from the interval $(2,3)$ should we choose?

How to select a single value: idea. A reasonable idea is to decrease the expected loss caused by an erroneous choice of $\alpha$. If we denote the loss caused by selecting a value $\widetilde{\alpha}$ when the actual value is $\alpha$ by $L(\widetilde{\alpha}, \alpha)$, then minimizing the expected loss means minimizing the expression $\int L(\widetilde{\alpha}, \alpha) \cdot f(\alpha) d \alpha$.

How to describe the corresponding loss function? The loss happens every time the selected value $\widetilde{\alpha}$ is different from 
the actual value $\alpha$, i.e., when the difference $\Delta \alpha \stackrel{\text { def }}{=} \widetilde{\alpha}-\alpha$ is different from 0 . Thus, it makes sense to consider a loss function which depends on this difference: $L(\widetilde{\alpha}, \alpha)=\ell(\Delta \alpha)$ for an appropriate function $\ell(x)$.

Which function $\ell(x)$ should we choose? It is reasonable to assume that the difference $\Delta \alpha$ is small, so we can expand the dependence $\ell(x)$ in Taylor series and keep the first few terms in this expansion:

$$
\ell(x)=\ell_{0}+\ell_{1} \cdot x+\ell_{2} \cdot x^{2}+\ldots
$$

When we selected $\alpha$ correctly, i.e., when $\Delta \alpha=0$, we should not have any loss, so we should have $\ell(0)=0$. Thus, we have $\ell_{0}=0$, and hence,

$$
\ell(x)=\ell_{1} \cdot x+\ell_{2} \cdot x^{2} .
$$

The loss is the smallest when $\Delta \alpha=0$. So, the function $\ell(x)$ attains its minimum for $x=0$. Thus, the derivative of the function $\ell(x)$ should be equal to 0 when $x=0$. This implies that $\ell_{1}=0$. Thus, the loss function is proportional to $(\Delta \alpha)^{2}$. So, the expected loss is equal to

$$
\ell_{2} \cdot \int_{2}^{3}(\widetilde{\alpha}-\alpha)^{2} \cdot f(\alpha) d \alpha
$$

For this loss function, which value $\alpha$ should we choose? Minimizing the expected loss means selecting a value $\widetilde{\alpha}$ for which the above expression attains the smallest possible value. Differentiating this expression with respect to the unknown $\widetilde{\alpha}$ and equating the derivative to 0 , we conclude that

$$
2 \ell_{2} \cdot\left(\widetilde{\alpha}-\int_{2}^{3} \alpha \cdot f(\alpha) d \alpha\right)=0,
$$

i.e., that'

$$
\widetilde{\alpha}=\int_{2}^{3} \alpha \cdot f(\alpha) d \alpha .
$$

For the uniform distribution $f(\alpha)=1$, this implies

$$
\widetilde{\alpha}=\int_{2}^{3} \alpha d \alpha=\left.\frac{\alpha^{2}}{2}\right|_{2} ^{3}=\frac{3^{2}}{2}-\frac{2^{2}}{2}=\frac{9-4}{2}=2.5 .
$$

Conclusion. So, in the probabilistic approach, we also arrive at the conclusion that the best value to choose is the value $\alpha=2.5$ - exactly the same value that we have empirically observed.

\section{CONCLUSiOns}

A recent statistical analysis of wars and terrorist attacks throughout the globe has shown that they follow a power law with exponent 2.5. Namely, the number of wars and terrorist attacks with $n$ casualties decreases with $n$ as $c \cdot n^{-\alpha}$ with $\alpha \approx 2.5$.
In this paper, we provide a theoretical explanation for this empirical phenomenon. First, we use general commonsense ideas to show that the corresponding exponent $\alpha$ should be located on the interval $(2,3)$. Then, we use fuzzy logic to conclude that out of all possible values from this interval, the most probable value is indeed $\alpha=2.5$.

This derivation was based on specific fuzzy techniques. A natural question is then: to what extent this derivation depends on these specific choices? Would our conclusion change if we used different fuzzy techniques or, more generally, a different approach to uncertainty? To answer this question, we analyzed the same problem from the viewpoint of a different uncertainty technique - namely, from the probabilistic viewpoint. To our pleasant surprise, it turned out that the probabilistic approach leads to the exact same value $\alpha=2.5$ for the desired exponent.

The fact that two different techniques for describing uncertainty lead to the same explanation makes us confident that this explanation is indeed correct.

\section{ACKNOWLEDGMENTS}

We acknowledge the partial support of the Chiang Mai University, Thailand, especially of the Center of Excellence in Econometrics, Faculty of Economics, Chiang Mai University.

This work was also partially supported by the National Science Foundation grants HRD-0734825 and HRD-1242122 (Cyber-ShARE Center of Excellence) and DUE-0926721, and by an award "UTEP and Prudential Actuarial Science Academy and Pipeline Initiative" from Prudential Foundation.

The authors are greatly thankful to the anonymous referees for valuable suggestions.

\section{REFERENCES}

[1] E. T. Jaynes and G. L. Bretthorst, Probability Theory: The Logic of Science, Cambridge University Press, Cambridge, UK, 2003.

[2] N. F. Johnson et al., "New online ecology of adversarial aggregates: ISIS and beyond", Science, 2016, Vol. 352, No. 6292, pp. 1459-1463.

[3] G. Klir and B. Yuan, Fuzzy Sets and Fuzzy Logic, Prentice Hall, Upper Saddle River, New Jersey, 1995.

[4] H. T. Nguyen and E. A. Walker, A First Course in Fuzzy Logic, Chapman and Hall/CRC, Boca Raton, Florida, 2006.

[5] D. Roberts and D. Turcotte, "Fractality and self-organized criticality of wars", Fractals: Complex Geometry, Patterns, and Scaling in Nature and Society, 1998. Vol. 6, No. 4, pp. 351-357.

[6] D. J. Sheskin, Handbook of Parametric and Nonparametric Statistical Procedures, Chapman and Hall/CRC, Boca Raton, Florida, 2011.

[7] L. A. Zadeh, "Fuzzy sets", Information and Control, 1965, Vol. 8, pp. 338-353. 